\title{
An Inventory Model for Ramp-Type Demand with Two-Level Trade Credit Financing Linked to Order Quantity
}

\author{
Hui-Ling Yang \\ Department of Computer Science and Information Engineering, Hungkuang University, Taiwan \\ Email: hui@sunrise.hk.edu.tw
}

How to cite this paper: Yang, H.-L. (2019) An Inventory Model for Ramp-Type Demand with Two-Level Trade Credit Financing Linked to Order Quantity. Open Journal of Business and Management, 7 , 427-446.

https://doi.org/10.4236/ojbm.2019.72029

Received: January 31, 2019

Accepted: March 4, 2019

Published: March 7, 2019

Copyright () 2019 by author(s) and Scientific Research Publishing Inc. This work is licensed under the Creative Commons Attribution International License (CC BY 4.0).

http://creativecommons.org/licenses/by/4.0/

\begin{abstract}
In the traditional economic order quantity (EOQ) model, it is assumed that the demand rate is constant. Thereafter, many researchers developed inventory model with time-varying demand to reflect sales in different phases of product life cycle in the market. However, in practice, especially for fashionable and high-tech product, the demand rate during the growth stages of its life cycle increases significantly with linear or exponential in the growth stage and then gradually stabilizes, and remains near constant in the maturity stage. It can be taken a ramp-type demand rate into account. Furthermore, in today's supply chain, a supplier usually offers a permissible delay in payment to retailers to encourage them to buy more products, and a retailer in turn provides a downstream trade-credit period to its customers. Therefore, this paper focus on 1) ramp-type demand rate and 2) the upstream and downstream trade credit financing linked to order quantity for retailer is considered. The objective is to find the optimal replenishment cycle and order quantity to keep the total relevant cost per unit time as minimum as possible. The study shows that in each case discussed, the optimal solution not only exists but also is unique. Numerical examples are provided to illustrate the proposed model. Finally, some relevant managerial insights based on the results are characterized.
\end{abstract}

\section{Keywords}

Ramp-Type Demand, Two-Level, Trade Credit, Finance, Order Quantity

\section{Introduction}

\subsection{Inventory Models with Ramp-Type Demand Rate}

In present, high-tech manufacturing is the backbone to the Taiwan economy. 
During the growth and maturity stages of a high-tech product life cycle, the demand rate increases significantly with linear or exponential in the growth stage and then remains near constant in the maturity stage. The term "ramp type" is used to represent such a demand pattern. Wu [1] developed an EOQ inventory model for Weibull distribution deterioration items with ramp type demand rate and partial backlogging. Manna and Chaudhuri [2] also developed an EOQ model with ramp type demand rate in which time dependent deterioration rate and shortages were considered. Agrawal et al. [3] provided an inventory model with deteriorating items, ramp-type demand and partially backlogged shortages for a two-warehouse system. Other related research articles on this field can be found in Deng [4], Deng et al. [5], Panda et al. [6] [7], Skouri et al. [8] [9] and their references.

\subsection{Inventory Models with Permissible Delay in Payment}

The traditional inventory economic order quantity (EOQ) model assumes that a buyer must pay for items immediately after receiving them. However, to stimulate sales quantity a supplier often offers a retailer a permissible delay in payment. Thus, to offer a certain fixed credit period for his/her retailer is an alternative incentive policy to quantity discount. In early research work, Goyal [10] developed an EOQ model under conditions of permissible delay in payments, and ignored the difference between the selling price and the purchase cost. Shah [11] considered a stochastic inventory model when delays in payments are permissible. Aggarwal and Jaggi [12] extended Goyal's model to consider the deteriorating items. Jamal et al. [13] further generalized Aggarwal and Jaggi's model to allow for shortages. Teng [14] amended Goyal's model by considering the difference between unit price and unit cost, and found that it makes economic sense for a well-established buyer to order less quantity and take the benefits of the permissible delay more frequently. Skouri et al. [15] proposed an inventory model with ramp type demand rate under permissible delay in payment. Teng et al. [16] established an economic order quantity model with trade credit financing for non-decreasing demand. Similarly, there are also many related articles published in such field with different practical consideration.

\subsection{Inventory Models with Two-Level Trade Credit}

Huang [17] extended Goyal's model to develop an EOQ model in which the supplier offers the retailer a permissible delay period (i.e., an upstream trade credit), and the retailer in turn provides a trade credit period (i.e., a downstream trade credit) to its customers. Teng and Goyal [18] complemented the shortcoming of Huang's model and proposed a generalized formulation. Teng et al. [19] obtained the retailer's optimal ordering policy when the supplier offers a progressive permissible delay in payments. Chen and Teng [20] provided a retailer's optimal ordering policy for deteriorating items with maximum lifetime under supplier's trade credit. Cheng and Teng [21] proposed an inventory and 
credit decision for time-varying deteriorating items with up-stream and downstream trade credit financing by discounted cash flow analysis. Shah [22] provided a three-layered integrated inventory model for deteriorating items with quadratic demand and two-level trade credit financing. Rameswari and Uthayakumar [23] proposed an integrated inventory model for deteriorating items with price-dependent demand under two-level trade credit policy. Several related articles can be found in Goyal et al. [24], Huang and Hsu [25], Min et al. [26] and their references.

\subsection{Inventory Models with Trade Credit Linked to Order Quantity}

Sometimes, to encourage more sales, supplier offer retailers a trade credit period with conditional permission, if a retailer orders more than a predetermined quantity. Chang et al. [27] developed an EOQ model for deteriorating items under supplier credits linked to ordering quantity. Chung and Liao [28] provided lot-sizing decisions under trade credit depending on the ordering quantity. Ouyang et al. [29] proposed an economic order quantity for deteriorating items with partially permissible delay in payments linked to order quantity. Kreng and Tan [30] proposed an inventory model under two levels of trade credit depending on the order quantity. Teng et al. [31] provided an inventory model for increasing demand under two levels of trade credit linked to order quantity, Recently, Sash and Cardenas-Barrón [32] provided an inventory model which is a retailer's decision for ordering and credit policies with deteriorating items when a supplier offers order-linked credit or cash discount. Ting [33] provided some comments on the EOQ model for deteriorating items with conditional trade credit linked to order quantity. Similarly, other related research articles can be found in their references.

In contrast to the above papers mentioned, this paper is extended in the following two ways: 1) a constant demand (or increasing demand) is extended to a ramp-type demand function, in which the demand increases linearly and then stays constant at the end, and 2) the supplier provides its retailer with a permissible delay link to order quantity while the retailer also offers a downstream trade credit period to its customers. We establish several fundamental theoretical results and obtain its optimal solution. We then provide several numerical examples to illustrate the proposed model and present some important and relevant managerial insights.

The rest of the paper is structured as follows. Section 2 introduces the notation and assumption needed to develop the proposed inventory model. Section 3 formulates the model. Section 4 discusses some theoretical results and provides an algorithm to find the optimal solutions. Section 5 provides numerical examples to illustrate the proposed model. Section 6 concludes the results and presents some managerial insights. Further, provides some future research directions.

\section{Notation and Assumptions}

The mathematical model of the inventory problem here is based on the follow- 
ing notation and assumptions:

\subsection{Notation}

The following notation is used throughout this paper.

$D(t)=$ the demand rate at time $t$, we here assume that $D(t)$ is deterministic at a constant rate after a period of time $\mu$, at initial the demand rate is increasing. i.e.,

$$
D(t)=\left\{\begin{array}{ll}
f(t), & t<\mu \\
f(\mu), & t \geq \mu
\end{array}, \text { where } f(t)=a+b t, a>0, \quad b>0 .\right.
$$

$Q_{d}=$ the minimum order quantity at which the delay is permitted by the supplier.

$T_{d}=$ the time interval that $Q_{d}$ units are depleted to zero due to demand.

$M=$ the retailer's upstream credit period offered by supplier in years.

$N=$ the retailer's downstream credit period offered to its buyers in years.

$T=$ the length of replenishment cycle in years.

$Q=$ the order quantity.

$A=$ the replenishment cost per order.

$h=$ the holding cost per unit per unit of time excluding interest charge.

$p=$ the unit selling price.

$c=$ the unit purchasing cost.

$I_{e}=$ the interest earned per dollar per year by the retailer.

$I_{p}=$ the interest paid per dollar per year by the retailer.

$I(t)=$ the inventory level at time $t$.

$T C_{i j}(T)=$ the total relevant cost per unit time for Case $i$ and subcase $j$, $i=1,2,3,4$ and $j=1,2$ or 3 , which is a function of $T$.

$T_{i j}^{*}=$ the optimal replenishment cycle time of $T C_{i j}(T)$ for Case $i$ and subcase $j, i=1,2,3,4$ and $j=1,2$ or 3. (i.e., $T^{*}$ ).

$Q_{i j}^{*}=$ the optimal order quantity for Case $i$ and subcase $j, i=1,2,3,4$ and $j=1,2$ or 3. (i.e., $Q^{*}$ ).

\subsection{Assumptions}

Next, the following assumptions are made to establish the mathematical inventory model.

1) Replenishment rate is instantaneous.

2) Shortages are not allowed to occur.

3) In today's global competition, many retailers have no pricing power. As a result, the selling price is hardly changed for many retailers. In addition, to avoid lasting price competition, we may assume without loss of generality that the selling price is constant in today's global competition and low inflation environment.

4) The objective here is to minimize the total relevant cost per unit of time until the demand is no longer increasing.

5) When $M-N>0$, the buyer deposits sales revenue into an interest bearing 
account from time $N$ to $M$. At the end of the permissible delay, the buyer pays off all units sold by time $M-N$, uses uncollected and unsold items as collateral to apply for a loan, and pays the bank a certain amount of money periodically until the loan is paid off at time $T+N$.

\section{Mathematical Formulation}

Based on the above assumptions, the inventory system here is as follows. At the beginning (i.e., at time $t=0$ ), the retailer orders and receives $Q$ units of a single product from the supplier. The inventory level is depleted gradually in the interval $[0, T]$ due to increasing demand from customers. At time $t=T$, the inventory level reaches zero. Hence, the inventory level at time $t, I(t)$, can be described by the following differential equation:

$$
\begin{aligned}
& \frac{\mathrm{d} I(t)}{\mathrm{d} t}=-f(t), \quad 0 \leq t \leq \mu \\
& \frac{\mathrm{d} I(t)}{\mathrm{d} t}=-f(\mu), \quad \mu \leq t \leq T
\end{aligned}
$$

with the boundary condition $I(T)=0$. The solution to (1) is

$$
\begin{aligned}
I(t) & =a(\mu-t)+b\left(\mu^{2}-t^{2}\right) / 2+(a+b \mu)(T-\mu), \quad 0 \leq t \leq \mu \\
& =(a+b \mu)(T-t), \quad \mu \leq t \leq T
\end{aligned}
$$

Thus, the retailer's order quantity per cycle is

$$
Q=I(0)=a \mu+b \mu^{2} / 2+(a+b \mu)(T-\mu)
$$

From Equation (3), we can obtain the time interval $T_{d}$ by using the following equations:

$$
Q_{d}=a \mu+b \mu^{2} / 2+(a+b \mu)\left(T_{d}-\mu\right)
$$

Next, based on whether the order quantity larger than the predetermined quantity or not, we have the following two cases: 1) $T<T_{d}$ 2) $T \geq T_{d}$.

\section{1. $Q<Q_{d}$ (i.e., $\left.T<T_{d}\right)$}

In this case, the retailer's order quantity is less than $Q_{d}$. Hence, the permissible delay in payment is not allowed (i.e., $M=0$ ). Meanwhile, the retailer offers a permissible delay of $N$ to its buyers. Consequently, the retailer must fiancé all items ordered at time 0 , and start to payoff the loan after time $N$. For details, please see Figure 1. Thus, the interest paid by the retailer is as follows. There are two cases to be discussed. 1) $0<\mu<N<T$ 2) $0<N<\mu<T$

$$
\begin{array}{cl}
c I_{p}\left[\int_{0}^{N} I(0) \mathrm{d} t+\int_{N}^{T+N} I(t-N) \mathrm{d} t\right] & \\
= \begin{cases}c I_{p}\left[\int_{0}^{\mu} I(0) \mathrm{d} t+\int_{\mu}^{N} I(0) \mathrm{d} t+\int_{N}^{T+N} I(t-N) \mathrm{d} t\right], & \text { if } 0<\mu<N<T \\
c I_{p}\left[\int_{0}^{N} I(0) \mathrm{d} t+\int_{N}^{\mu} I(t-N) \mathrm{d} t+\int_{\mu}^{T+N} I(t-N) \mathrm{d} t\right], & \text { if } 0<N<\mu<T\end{cases}
\end{array}
$$

Hence, the retailer's total relevant cost per unit time is 


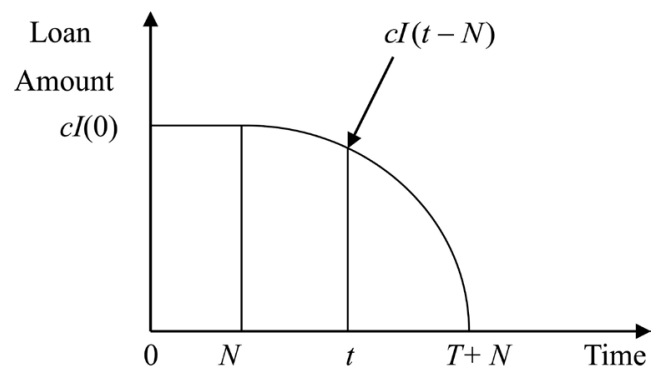

Figure 1. Graphical representation for $T<T_{d}$.

$$
\begin{aligned}
& T C_{11}(T)\left\{A+h \int_{0}^{T} I(t) \mathrm{d} t+c I_{p}\left[\int_{0}^{\mu} I(0) \mathrm{d} t+\int_{\mu}^{N} I(0) \mathrm{d} t+\int_{N}^{T+N} I(t-N) \mathrm{d} t\right]\right\} / T \\
&=\left\{A+h\left[\frac{a \mu^{2}}{2}+\frac{b \mu^{3}}{3}+\frac{\left.(a+b \mu)\left(T^{2}-\mu^{2}\right)\right]}{2}\right]\right. \\
&\left.+c I_{p}\left[\left(a \mu+\frac{b \mu^{2}}{2}\right) \mu+(a+b \mu)\left(T N-\mu^{2}\right)+\frac{a+b \mu}{2} T^{2}\right]\right\} / T, \text { if } 0<\mu<N<T \\
& T C_{12}(T) \\
&=\left\{A+h \int_{0}^{T} I(t) \mathrm{d} t+c I_{p}\left[\int_{0}^{N} I(0) \mathrm{d} t+\int_{N}^{\mu} I(t-N) \mathrm{d} t+\int_{\mu}^{T+N} I(t-N) \mathrm{d} t\right]\right\} / T \\
&=\left\{A+h\left[\frac{a \mu^{2}}{2}+\frac{b \mu^{3}}{3}+\frac{(a+b \mu)\left(T^{2}-\mu^{2}\right)}{2}\right]\right. \\
&+c I_{p}\left[\left(a \mu+\frac{b \mu^{2}}{2}\right) N+\frac{\mu-N}{6}\left(3 a(\mu+N)+b\left(2 \mu^{2}+2 \mu N-N^{2}\right)\right)\right. \\
&+\left.\left.\frac{a+b \mu}{2}\left(T^{2}-\mu^{2}+N^{2}\right)\right]\right\} / T, \quad \text { if } 0<N<\mu<T
\end{aligned}
$$

\section{2. $Q \geq Q_{d}$ (i.e., $T \geq T_{d}$ )}

In this case, based on the supplier's trade credit $M$, and the last customer's payment time $T+N$, we discuss the following three cases: 1) $0<M<N \quad$ 2) $M \geq N$ and $M \leq T+N$ 3) $M \geq N$ and $M>T+N$.

\subsubsection{The Case of $0<M<N$}

Since $0<M<N$, there is no interest earned for the retailer. In addition, the retailer has to finance all items ordered after time $M$ at an interest charged $I_{p}$ per dollar per year, and start to pay off the loan after time $N$ as shown in Figure 2. Consequently, the interest charged is given by

$$
\begin{array}{ll}
c I_{p}\left[\int_{M}^{N} I(0) \mathrm{d} t+\int_{N}^{T+N} I(t-N) \mathrm{d} t\right] & \\
= \begin{cases}c I_{p}\left[\int_{M}^{N} I(0) \mathrm{d} t+\int_{N}^{T+N} I(t-N) \mathrm{d} t\right], & \text { if } 0<\mu<M<N<T \\
c I_{p}\left[\int_{M}^{\mu} I(0) \mathrm{d} t+\int_{\mu}^{N} I(0) \mathrm{d} t+\int_{N}^{T+N} I(t-N) \mathrm{d} t\right], & \text { if } 0<M<\mu<N<T \\
c I_{p}\left[\int_{M}^{N} I(0) \mathrm{d} t+\int_{N}^{\mu} I(t-N) \mathrm{d} t+\int_{\mu}^{T+N} I(t-N) \mathrm{d} t\right], & \text { if } 0<M<N<\mu<T\end{cases}
\end{array}
$$




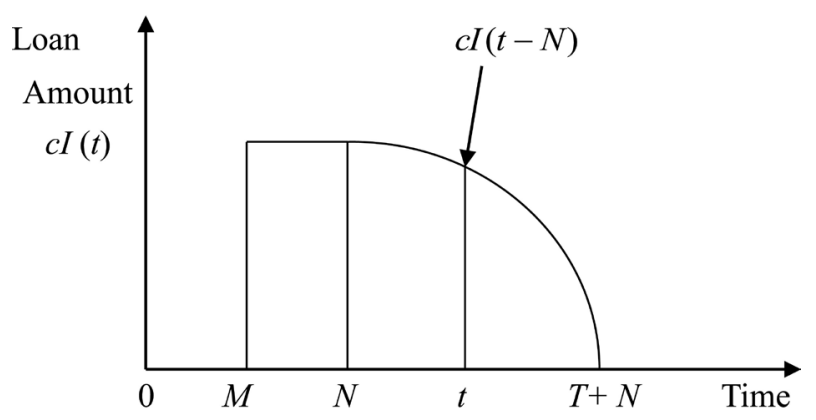

Figure 2. Graphical representation for $T \geq T_{d}$ and $M<N$.

Hence, the retailer's total relevant cost per unit time is

$$
\begin{aligned}
& T C_{21}(T) \\
= & \left\{A+h \int_{0}^{T} I(t) \mathrm{d} t+c I_{p}\left[\int_{M}^{N} I(0) \mathrm{d} t+\int_{N}^{T+N} I(t-N) \mathrm{d} t\right]\right\} / T \\
= & \left\{A+h\left[\frac{a \mu^{2}}{2}+\frac{b \mu^{3}}{3}+\frac{(a+b \mu)\left(T^{2}-\mu^{2}\right)}{2}\right]\right. \\
& \left.+c I_{p}(a+b \mu)\left[T(N-M)+\frac{T^{2}}{2}\right]\right\} / T, \text { if } 0<\mu<M<N<T
\end{aligned}
$$

$T C_{22}(T)$

$=\left\{A+h \int_{0}^{T} I(t) \mathrm{d} t+c I_{p}\left[\int_{M}^{\mu} I(0) \mathrm{d} t+\int_{\mu}^{N} I(0) \mathrm{d} t+\int_{N}^{T+N} I(t-N) \mathrm{d} t\right]\right\} / T$

$=\left\{A+h\left[\frac{a \mu^{2}}{2}+\frac{b \mu^{3}}{3}+\frac{(a+b \mu)\left(T^{2}-\mu^{2}\right)}{2}\right]+c I_{p}\left[\left(a \mu+\frac{b \mu^{2}}{2}\right)(\mu-M)\right.\right.$

$$
\left.\left.+(a+b \mu)\left(T(N-M)+\mu(M-\mu)+\frac{T^{2}}{2}\right)\right]\right\} / T, \text { if } 0<M<\mu<N<T
$$

$T C_{23}(T)$

$=\left\{A+h \int_{0}^{T} I(t) \mathrm{d} t+c I_{p}\left[\int_{M}^{N} I(0) \mathrm{d} t+\int_{N}^{\mu} I(0) \mathrm{d} t+\int_{\mu}^{T+N} I(t-N) \mathrm{d} t\right]\right\} / T$

$=\left\{A+h\left[\frac{a \mu^{2}}{2}+\frac{b \mu^{3}}{3}+\frac{(a+b \mu)\left(T^{2}-\mu^{2}\right)}{2}\right]+c I_{p}\left[\left(a \mu+\frac{b \mu^{2}}{2}\right)(N-M)\right.\right.$

$+\frac{\mu-N}{6}\left(3 a(\mu+N)+b\left(2 \mu^{2}+2 \mu N-N^{2}\right)\right)$

$\left.\left.+\frac{a+b \mu}{2}\left((T-\mu)(T+\mu+2 N-2 M)+N^{2}\right)\right]\right\} / T$, if $0<M<N<\mu<T$

\subsubsection{The Case of $M \geq N$ and $M \leq T+N$}

When $M \leq T+N$, the retailer cannot receive the last payment before the permissible delay time $M$. As a result, the retailer must finance all items sold after time $(M-N)$ at time $M$, and pay off the loan until $T+N$ at an interest rate of $I_{p}$ per dollar per year as shown in Figure 3. Therefore, the interest paid is given by 


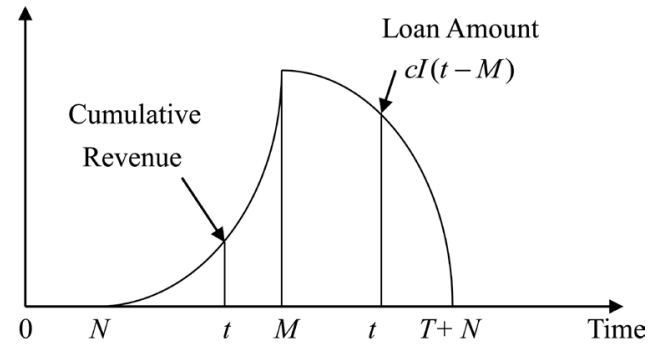

Figure 3. Graphical representation for $T \geq T_{d}, \quad 0<N \leq M$ and $M \leq T+N$.

$$
\begin{aligned}
& c I_{p} \int_{M}^{T+N} I(t-M) \mathrm{d} t \\
& = \begin{cases}c I_{p} \int_{M}^{T+N} \int_{t-M}^{T} f(\mu) \mathrm{d} v \mathrm{~d} t, & \text { if } \mu \leq M \\
c I_{p} \int_{M}^{T+N}\left(\int_{t-M}^{\mu} f(v) \mathrm{d} v+\int_{\mu}^{T} f(\mu) \mathrm{d} v\right) \mathrm{d} t, & \text { if } M<\mu\end{cases}
\end{aligned}
$$

On the other hand, the retailer starts selling products at time 0 , and receiving the money at time $N$. Consequently, the retailer accumulates sales revenue in an account that earns $I_{e}$ per dollar per year starting from $N$ through $M$ as shown in Figure 3. Therefore, the interest earned is given by

$$
\begin{aligned}
& p I_{e} \int_{N}^{M} \int_{0}^{t-N} f(v) \mathrm{d} v \mathrm{~d} t \\
& = \begin{cases}p I_{e} \int_{N}^{M}\left(\int_{0}^{\mu} f(v) \mathrm{d} v+\int_{\mu}^{t-N} f(\mu) \mathrm{d} v\right) \mathrm{d} t, & \text { if } \mu \leq N \\
p I_{e} \int_{N}^{M}\left(\int_{0}^{N} f(v) \mathrm{d} v+\int_{N}^{\mu} f(v-N) \mathrm{d} v+\int_{\mu}^{t-N} f(\mu) \mathrm{d} v\right) \mathrm{d} t, & \text { if } N<\mu\end{cases}
\end{aligned}
$$

As a result, the retailer's total relevant cost per unit time is

$$
\begin{aligned}
& T C_{31}(T)=\left\{A+h \int_{0}^{T} I(t) \mathrm{d} t+c I_{p} \int_{M}^{T+N} \int_{t-M}^{T} f(\mu) \mathrm{d} v \mathrm{~d} t\right. \\
& \left.\quad-p I_{e} \int_{N}^{M}\left(\int_{0}^{\mu} f(v) \mathrm{d} v+\int_{\mu}^{t-N} f(\mu) \mathrm{d} v\right) \mathrm{d} t\right\} / T \\
& =\left\{A+h\left[\frac{a \mu^{2}}{2}+\frac{b \mu^{3}}{3}+\frac{(a+b \mu)\left(T^{2}-\mu^{2}\right)}{2}\right]\right. \\
& +c I_{p}(a+b \mu)\left[T^{2}-(M-N)^{2}\right] / 2-p I_{e}\left[\left(a \mu+\frac{b \mu^{2}}{2}\right)(M-N)\right. \\
& \left.\left.+(a+b \mu)\left((M-N-\mu)(M-N)-\frac{(M-N)^{2}}{2}\right)\right]\right\} / T, \text { if } 0<\mu \leq N<M \\
& T C_{32}(T)=\left\{A+h \int_{0}^{T} I(t) \mathrm{d} t+c I_{p} \int_{M}^{T+N} \int_{t-M}^{T} f(\mu) \mathrm{d} v \mathrm{~d} t\right. \\
& \left.\quad-p I_{e} \int_{N}^{M}\left(\int_{0}^{N} f(v) \mathrm{d} v+\int_{N}^{\mu} f(v-N) \mathrm{d} v+\int_{\mu}^{t-N} f(\mu) \mathrm{d} v\right) \mathrm{d} t\right\} / T \\
& =\left\{\begin{array}{c}
A \\
A
\end{array}\left[\frac{a \mu^{2}}{2}+\frac{b \mu^{3}}{3}+\frac{(a+b \mu)\left(T^{2}-\mu^{2}\right)}{2}\right]+c I_{p}(a+b \mu)\left[T^{2}-(M-N)^{2}\right] / 2\right. \\
& -p I_{e}\left[\left(a \mu+\frac{b \mu^{2}}{2}\right)(M-N)-b N(\mu-N)(M-N)\right. \\
& \left.\left.+(a+b \mu)\left((M-N-\mu)(M-N)-\frac{(M-N)^{2}}{2}\right)\right]\right\} / T, \text { if } 0<N<\mu<M
\end{aligned}
$$




$$
\begin{aligned}
& T C_{33}(T)=\left\{A+h \int_{0}^{T} I(t) \mathrm{d} t+c I_{p} \int_{M}^{T+N}\left(\int_{t-M}^{\mu} f(v) \mathrm{d} v+\int_{\mu}^{T} f(\mu) \mathrm{d} v\right) \mathrm{d} t\right. \\
& \left.-p I_{e} \int_{N}^{M}\left(\int_{0}^{\mu-N} f(v) \mathrm{d} v+\int_{\mu-N}^{t-N} f(\mu) \mathrm{d} v\right) \mathrm{d} t\right\} / T \\
& =\left\{A+h\left[\frac{a \mu^{2}}{2}+\frac{b \mu^{3}}{3}+\frac{(a+b \mu)\left(T^{2}-\mu^{2}\right)}{2}\right]\right. \\
& +c I_{p}\left[\frac{a}{2}\left(\mu^{2}-(M+\mu-T-N)^{2}\right)+\frac{b}{2}\left(\mu^{2}(T+N-M)-\frac{(T+N-M)^{3}}{3}\right)\right. \\
& -p I_{e}\left[\left(a \mu+\frac{b \mu^{2}}{2}\right)(M-N)-b N(\mu-N)(M-N)\right. \\
& \left.\left.+(a+b \mu)\left((M-N-\mu)(M-N)-\frac{(M-N)^{2}}{2}\right)\right]\right\} / T, \text { if } 0<N<M<\mu
\end{aligned}
$$

\subsubsection{The Case of $M \geq N$ and $M>T+N$}

Since the order quantity is larger than or equal to $Q_{d}$ (due to $T_{d} \leq T$ ), the retailer receives the permissible delay in payment. If $T+N<M$, then the retailer receives all payments from its customers by the time $T+N$ which is before the permissible delay time $M$. Hence, the retailer has the money to pay the supplier at time $M$, and does not have the interest charges. In the meantime, the retailer receives the revenue and deposits into a bank to earn interest as shown in Figure 4. The interest earned by the retailer is

$$
\begin{aligned}
& p I_{e}\left[\int_{N}^{T+N}[I(0)-I(t-N)] \mathrm{d} t+\int_{T+N}^{M} I(0) \mathrm{d} t\right] \\
& =p I_{e}\left[\int_{N}^{T+N} \int_{0}^{t-N} f(v) \mathrm{d} v \mathrm{~d} t+\int_{T+N}^{M} I(0) \mathrm{d} t\right] \\
& = \begin{cases}p I_{e}\left[\int_{N}^{T+N}\left(\int_{0}^{\mu} f(v) \mathrm{d} v+\int_{\mu}^{t-N} f(\mu) \mathrm{d} v\right) \mathrm{d} t+\int_{T+N}^{M} I(0) \mathrm{d} t\right], & \text { if } \mu<N<M \\
p I_{e}\left[\int_{N}^{T+N}\left(\int_{0}^{N} f(v) \mathrm{d} v+\int_{N}^{\mu} f(v-N) \mathrm{d} v+\int_{\mu}^{t-N} f(\mu) \mathrm{d} v\right) \mathrm{d} t+\int_{T+N}^{M} I(0) \mathrm{d} t\right], & \text { if } N<\mu<M\end{cases}
\end{aligned}
$$

Hence, the retailer's total relevant cost per unit time is

$$
\begin{gathered}
T C_{41}(T)=\left\{A+h \int_{0}^{T} I(t) \mathrm{d} t-p I_{e}\left[\int_{N}^{T+N}\left(\int_{0}^{\mu} f(v) \mathrm{d} v+\int_{\mu}^{t-N} f(\mu) \mathrm{d} v\right) \mathrm{d} t\right.\right. \\
\left.\left.+\int_{T+N}^{M} I(0) \mathrm{d} t\right]\right\} / T \\
=\left\{A+h\left[\frac{a \mu^{2}}{2}+\frac{b \mu^{3}}{3}+\frac{(a+b \mu)\left(T^{2}-\mu^{2}\right)}{2}\right]-p I_{e}\left[\left(a \mu+\frac{b \mu^{2}}{2}\right)(M-N)\right.\right. \\
\left.\left.+(a+b \mu)\left((T-\mu)(M-N)-\frac{T^{2}}{2}\right)\right]\right\} / T, \text { if } 0<\mu<N<T+N<M \\
T C_{42}(T)=\left\{A+h \int_{0}^{T} I(t) \mathrm{d} t-p I_{e}\left[\int _ { N } ^ { T + N } \left(\int_{0}^{N} f(v) \mathrm{d} v+\int_{N}^{\mu} f(v-N) \mathrm{d} v\right.\right.\right. \\
\left.\left.\left.+\int_{\mu}^{t-N} f(\mu) \mathrm{d} v\right) \mathrm{~d} t+\int_{T+N}^{M} I(0) \mathrm{d} t\right]\right\} / T
\end{gathered}
$$




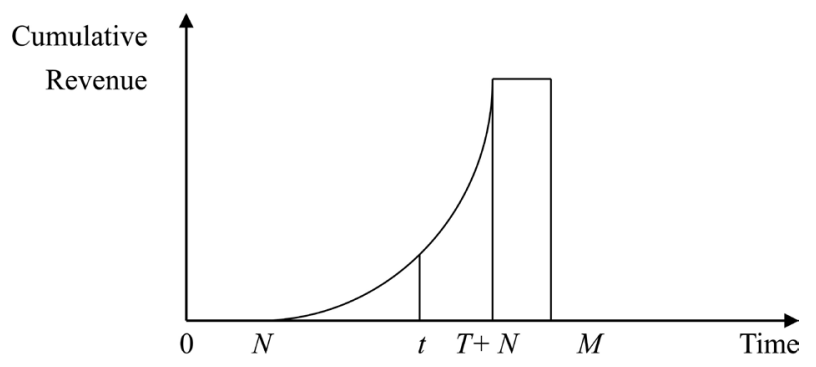

Figure 4. Graphical representation for $T \geq T_{d}, \quad 0<N \leq M$ and $M>T+N$.

$$
\begin{aligned}
= & \left\{A+h\left[\frac{a \mu^{2}}{2}+\frac{b \mu^{3}}{3}+\frac{(a+b \mu)\left(T^{2}-\mu^{2}\right)}{2}\right]\right. \\
& -p I_{e}\left[\left(a \mu+\frac{b \mu^{2}}{2}\right)(M-N)-b N(\mu-N) T\right. \\
& \left.\left.+(a+b \mu)\left((T-\mu)(M-N)-\frac{T^{2}}{2}\right)\right]\right\} / T, \text { if } 0<N<\mu<T+N<M .
\end{aligned}
$$

\section{Theoretical Results}

To minimize the total relevant cost, taking the first and second order derivatives of $T C_{i j}(T), \quad i=1,2,3,4, \quad j=1,2$ or 3 with respect to $T$ and let $\mathrm{d} T C_{i j}(T) / \mathrm{d} T=0$, we obtain the following results.

$$
\begin{aligned}
& \frac{\mathrm{d} T C_{11}}{\mathrm{~d} T}=\left\{h(a+b \mu) T+c I_{p}(a+b \mu)(T+N)-T C_{11}\right\} / T=0, \\
& \frac{\mathrm{d} T C_{12}}{\mathrm{~d} T}=\left\{h(a+b \mu) T+c I_{p}(a+b \mu) T-T C_{12}\right\} / T=0, \\
& \left.\frac{\mathrm{d}^{2} T C_{1 j}}{\mathrm{~d} T^{2}}\right|_{\frac{\mathrm{d} T C_{1 j}=0}{\mathrm{~d} T}=0}=\left(h+c I_{p}\right)(a+b \mu) / T>0, \quad j=1,2 . \\
& \frac{\mathrm{d} T C_{2 j}}{\mathrm{~d} T}=\left\{h(a+b \mu) T+c I_{p}(a+b \mu)(T+N-M)-T C_{2 j}\right\} / T=0, \quad j=1,2,3 . \\
& \left.\frac{\mathrm{d}^{2} T C_{2 j}}{\mathrm{~d} T^{2}}\right|_{\frac{\mathrm{d} T C_{2 j}}{\mathrm{~d} T}=0}=\left(h+c I_{p}\right)(a+b \mu) / T>0, j=1,2,3 . \\
& \frac{\mathrm{d} T C_{3 j}}{\mathrm{~d} T}=\left\{\left(h+c I_{p}\right)(a+b \mu) T-T C_{3 j}\right\} / T=0, \quad j=1,2 . \\
& \frac{\mathrm{d} T C_{33}}{\mathrm{~d} T}=\left\{h(a+b \mu) T+c I_{p}[a(\mu+M-T-N)\right. \\
& \left.\left.+\frac{b}{2}\left(\mu^{2}-(T+N-M)^{2}\right)\right]-T C_{33}\right\} / T \\
& =0 \\
& \left.\frac{\mathrm{d}^{2} T C_{3 j}}{\mathrm{~d} T^{2}}\right|_{\frac{\mathrm{d} T C_{3 j}}{\mathrm{~d} T}=0}=\left(h+c I_{p}\right)(a+b \mu) / T>0, j=1,2 .
\end{aligned}
$$




$$
\begin{gathered}
\left.\frac{\mathrm{d}^{2} T C_{33}}{\mathrm{~d} T^{2}}\right|_{\frac{\mathrm{d} T C_{33}}{\mathrm{~d} T}=0}=\left[h(a+b \mu)-c I_{p}(a+b(T+N-M))\right] / T>0 . \\
\frac{\mathrm{d} T C_{41}}{\mathrm{~d} T}=\left\{h(a+b \mu) T-p I_{e}(a+b \mu)(M-T-N)-T C_{41}\right\} / T=0, \\
\frac{\mathrm{d} T C_{42}}{\mathrm{~d} T}=\left\{h(a+b \mu) T-p I_{e}[-b N(N-\mu)+(a+b \mu)(M-T-N)]-T C_{42}\right\} / T=0, \\
\left.\frac{\mathrm{d}^{2} T C_{4 j}}{\mathrm{~d} T^{2}}\right|_{\frac{\mathrm{d} T C_{4 j}}{\mathrm{~d} T}=0}=\left(h+p I_{e}\right)(a+b \mu) / T>0, j=1,2 .
\end{gathered}
$$

Theorem 1. For each $i$, $j$, there exists a unique optimal cycle length $T_{i j}$ which minimizes $T C_{i j}, i=1,2,3,4, j=1,2$ or 3 .

\section{Proof: See Appendix.}

It is not easily to find the closed-form of $T$ from the equation of each first derivative which is equal to zero. However, we can use numerical method to find the solution. From Theorem 1, we know that the solution minimizes the total relevant cost function is also a global minimum. By ensuring the solution satisfies the condition in each case, the following theoretical result is obtained.

Corollary 1. For $Q<Q_{d}$,

(a) if $T_{11}<T_{d}$ and $0<\mu<N<T_{11}$, then $T^{*}=T_{11}$.

(b) if $T_{12}<T_{d}$ and $0<N<\mu<T_{12}$, then $T^{*}=T_{12}$

Corollary 2. For $Q \geq Q_{d}$,

(a) $0<M<N$,

(i) if $T_{21} \geq T_{d}$ and $0<\mu<M<N<T_{21}$, then $T^{*}=T_{21}$.

(ii) if $T_{22} \geq T_{d}$ and $0<M<\mu<N<T_{22}$, then $T^{*}=T_{22}$.

(iii) if $T_{23} \geq T_{d}$ and $0<M<N<\mu<T_{23}$, then $T^{*}=T_{23}$

(b) $0<N \leq M$ and $M \leq T+N$,

(i) if $T_{31} \geq T_{d}$ and $0<\mu \leq N<M \leq T_{31}+N$, then $T^{*}=T_{31}$.

(ii) if $T_{32} \geq T_{d}$ and $0<N<\mu<M \leq T_{32}+N$, then $T^{*}=T_{32}$.

(iii) if $T_{33} \geq T_{d}$ and $0<N<M<\mu \leq T_{33}+N$, then $T^{*}=T_{33}$.

(c) $0<N \leq M$ and $M>T+N$

(i) if $T_{41} \geq T_{d}$ and $0<\mu<N<T_{41}+N<M$, then $T^{*}=T_{41}$.

(ii) if $T_{42} \geq T_{d}$ and $0<N<\mu<T_{42}+N<M$, then $T^{*}=T_{42}$.

Summarizing the results in Corollary 1 and 2, we propose the following algorithm to find the optimal solution.

\section{Algorithm}

Step 0. Input parameter values.

Step 0.1. By (4), calculate $T_{d}$

Step 0.2. Compare the values of $M$ and $N$. If $M<N$, then go to Step 1 . Otherwise, go to Step 4.

Step 1. By (14.1), (14.2), (16), calculate $T$, let it be $T_{11}, T_{12}, T_{21}, T_{22}, T_{23}$.

Step 2. Compare the values of $T_{i j}, i=1,2, j=1,2$ or 3 , and $T_{d}$.

Step 2.1. If $T_{11}<T_{d}$ and $0<\mu<N<T_{11}$, then $T^{*}=T_{11}$, and calcu- 
late $T C_{11}\left(T^{*}\right)$. Otherwise, set $T C_{11}\left(T_{11}\right)=\infty$.

Step 2.2. If $T_{12}<T_{d}$ and $0<N<\mu<T_{12}$, then $T^{*}=T_{12}$, and calculate $T C_{12}\left(T^{*}\right)$. Otherwise, set $T C_{12}\left(T_{12}\right)=\infty$.

Step 2.3. If $T_{21} \geq T_{d}$ and $0<\mu<M<N<T_{21}$, then $T^{*}=T_{21}$ and calculate $T C_{21}\left(T^{*}\right)$, Otherwise, set $T C_{21}\left(T_{21}\right)=\infty$.

Step 2.4. If $T_{22} \geq T_{d}$ and $0<M<\mu<N<T_{22}$, then $T^{*}=T_{22}$, and calculate $T C_{22}\left(T^{*}\right)$. Otherwise, set $T C_{22}\left(T_{22}\right)=\infty$.

Step 2.5. If $T_{23} \geq T_{d}$ and $0<M<N<\mu<T_{23}$, then $T^{*}=T_{23}$ and calculate $T C_{23}\left(T^{*}\right)$. Otherwise, set $T C_{23}\left(T_{23}\right)=\infty$.

Step 3. Set $T C\left(T^{*}\right)=\min \left\{T C_{i j}\left(T_{i j}\right) \mid i=1,2, j=1,2\right.$ or 3$\}$, then $T^{*}=T_{i j}$ is the optimal solution, for a certain $i, j$ and stop.

Step 4. By (18.1), (18.2), (20.1), (20.2), calculate $T$, let it be $T_{31}, T_{32}, T_{33}, T_{41}$, $T_{42}$.

Step 5. Compare the values of $T_{i j}, i=3,4, j=1,2$ or 3, and $T_{d}$.

Step 5.1. If $T_{31} \geq T_{d}$ and $0<\mu \leq N<M \leq T_{31}+N$, then $T^{*}=T_{31}$ and calculate $T C_{31}\left(T^{*}\right)$. Otherwise, set $T C_{31}\left(T_{31}\right)=\infty$.

Step 5.2. If $T_{32} \geq T_{d}$ and $0<N<\mu<M \leq T_{32}+N$, then $T^{*}=T_{32}$ and calculate $T C_{32}\left(T^{*}\right)$, Otherwise, set $T C_{32}\left(T_{32}\right)=\infty$.

Step 5.3. If $T_{33} \geq T_{d}$ and $0<N<M<\mu \leq T_{33}+N$, then $T^{*}=T_{33}$ and calculate $T C_{33}\left(T^{*}\right)$, Otherwise, set $T C_{33}\left(T_{33}\right)=\infty$

Step 5.4. If $T_{41} \geq T_{d}$ and $0<\mu<N<T_{41}+N<M$, then $T^{*}=T_{41}$. and calculate $T C_{41}\left(T^{*}\right)$. Otherwise, set $T C_{41}\left(T_{41}\right)=\infty$.

Step 5.5. If $T_{42} \geq T_{d}$ and $0<N<\mu<T_{42}+N<M$, then $T^{*}=T_{42}$ and calculate $T C_{42}\left(T^{*}\right)$. Otherwise, set $T C_{42}\left(T_{42}\right)=\infty$.

Step 6. Set $T C\left(T^{*}\right)=\min \left\{T C_{i j}\left(T_{i j}\right) \mid i=3,4, j=1,2\right.$ or 3$\}$, then $T^{*}=T_{i j}$ is the optimal solution, for a certain $i, j$ and stop.

\section{Numerical Examples}

In this section, we provide two numerical examples to illustrate several distinct theoretical results for $M>N$ and $M<N$. Let the demand rate $f(t)=100+50 t$ per year, $A=\$ 10$ per order, $h=\$ 3 /$ unit/year, $c=\$ 5 /$ unit, $p=\$ 10 /$ unit, $I_{p}=$ $0.06 /$ year, and $I_{e}=0.05 /$ year.

\section{1. $M<N$}

Let $M=1 / 12$ years, and $N=1 / 6$ years.

1) Let $Q_{d}=30$ units.

Example 1.1. Let $\mu=0.1$ years, we know that $\mu<N$. By (4), we have $T_{d}=0.28810$ years and by the above algorithm, we have

$$
T_{11}=0.23986, T_{12}=0.24614, T_{21}=0.23995, T_{22}=0.23993, T_{23}=0.23998
$$

and

$$
T C_{11}\left(T_{11}\right)=88.36124, \quad T C_{12}\left(T_{12}\right)=\infty, \quad T C_{21}\left(T_{21}\right)=\infty, \quad T C_{22}\left(T_{22}\right)=\infty,
$$


$T C_{23}\left(T_{23}\right)=\infty$. Since $T_{11}<T_{d}$ and $\mu<N<T_{11}$, by Corollary 1 (a), we know that the optimal solution is $T^{*}=T_{11}=0.23986$ years, and then $T C\left(T^{*}\right)=T C_{11}\left(T_{11}\right)=88.36124$. Furthermore, by (3), we have $Q^{*}=Q_{11}=24.93522$ units.

Example 1.2. Let $\mu=0.2$ years, we know that $N<\mu$. By (4), we have $T_{d}=0.28182$ years and by the above algorithm, we have

$$
T_{11}=0.23165, T_{12}=0.24440, T_{21}=0.23237, T_{22}=0.23195, T_{23}=0.23197
$$

and

$$
T C_{11}\left(T_{11}\right)=\infty, T C_{12}\left(T_{12}\right)=88.71856, T C_{21}\left(T_{21}\right)=\infty, T C_{22}\left(T_{22}\right)=\infty,
$$

$T C_{23}\left(T_{23}\right)=\infty$. Since $T_{12}<T_{d}$ and $N<\mu<T_{12}$, by Corollary 1(b), we know that the optimal solution is $T^{*}=T_{12}=0.24440$ years, and then $T C\left(T^{*}\right)=T C_{12}\left(T_{12}\right)=88.71856$. By (3), we have $Q^{*}=Q_{12}=25.88441$ units.

2) Let $Q_{d}=20$ units.

Example 1.3. Let $\mu=0.05$ years, we know that $\mu<M<N$. By (4), we have $T_{d}=0.19573$ years and by the above algorithm, we have

$$
T_{11}=0.24311, T_{12}=0.24632, T_{21}=0.24312, T_{22}=0.24313, T_{23}=0.24324
$$

and

$$
T C_{11}\left(T_{11}\right)=\infty, T C_{12}\left(T_{12}\right)=\infty, T C_{21}\left(T_{21}\right)=84.79927, T C_{22}\left(T_{22}\right)=\infty,
$$

$T C_{23}\left(T_{23}\right)=\infty$. Since $T_{21}>T_{d}$ and $\mu<M<N<T_{21}$, by Corollary 2(a)(i), we know that the optimal solution is $T^{*}=T_{21}=0.24312$ years, and then $T C\left(T^{*}\right)=T C_{21}\left(T_{21}\right)=84.79927$. By (3), we have $Q^{*}=Q_{21}=24.85773$ units.

Example 1.4. Let $\mu=0.1$ years, we know that $M<\mu<N$. By (4), we have $T_{d}=0.19286$ years and by the above algorithm, we have

$$
T_{11}=0.23986, T_{12}=0.24614, T_{21}=0.23995, T_{22}=0.23993, T_{23}=0.23998
$$

and

$$
T C_{11}\left(T_{11}\right)=\infty, T C_{12}\left(T_{12}\right)=\infty, T C_{21}\left(T_{21}\right)=\infty, T C_{22}\left(T_{22}\right)=85.76229,
$$

$T C_{23}\left(T_{23}\right)=\infty$. Since $T_{22}>T_{d}$ and $M<\mu<N<T_{22}$, by Corollary 2(a)(ii), we know that the optimal solution is $T^{*}=T_{22}=0.24440$ years, and then $T C\left(T^{*}\right)=T C_{22}\left(T C_{22}\right)=85.76229$. By (3), we have $Q^{*}=Q_{22}=24.94312$ units.

Example 1.5. Let $\mu=0.2$ years, we know that $M<N<\mu$. By (4), we have $T_{d}=0.19091$ years and by the above algorithm, we have

$$
T_{11}=0.23165, T_{12}=0.24440, T_{21}=0.23237, T_{22}=0.23195, T_{23}=0.23197
$$

and

$$
T C_{11}\left(T_{11}\right)=\infty, T C_{12}\left(T_{12}\right)=\infty, T C_{21}\left(T_{21}\right)=\infty, T C_{22}\left(T_{22}\right)=\infty,
$$

$T C_{23}\left(T_{23}\right)=86.95530$.. Since $T_{23}>T_{d}$ and $M<N<\mu<T_{23}$, by Corollary 2(a) (iii), we know that the optimal solution is $T^{*}=T_{23}=0.23197$ years, and then $T C\left(T^{*}\right)=T C_{23}\left(T_{23}\right)=86.95530$. By (3), we have $Q^{*}=Q_{23}=24.51676$ units.

\section{2. $M \geq N$}

In this subsection, let $Q_{d}=20$ units,

1) Let $M=1 / 6$ years, and $N=1 / 12$ years. 
Example 2.1. Let $\mu=0.05$ year, we know that $\mu<N \leq M$. By (4), we have $T_{d}=0.19573$ years and by the above algorithm, we have

$$
T_{31}=0.23967, T_{32}=0.23960, T_{33}=0.26351, T_{41}=0.23611, T_{42}=0.23360
$$

and

$$
T C_{31}\left(T_{31}\right)=81.06808, T C_{32}\left(T_{32}\right)=\infty, T C_{33}\left(T_{33}\right)=\infty, T C_{41}\left(T_{41}\right)=\infty,
$$

$T C_{42}\left(T_{42}\right)=\infty$. Since $T_{31}>T_{d}$ and $\mu<N<M<T_{31}+N$, by Corollary 2(b)(i), we know that the optimal solution is $T^{*}=T_{31}=0.23967$ years, and then $T C\left(T^{*}\right)=T C_{31}\left(T_{31}\right)=81.06808$. By (3), we have $Q^{*}=Q_{31}=24.50358$ units.

Example 2.2. Let $\mu=0.1$ years, we know that $N<\mu<M$. By (4), we have $T_{d}=0.19286$ years and by the above algorithm, we have

$$
T_{31}=0.23654, T_{32}=0.23658, T_{33}=0.25808, T_{41}=0.23311, T_{42}=0.23435
$$

and

$$
T C_{31}\left(T_{31}\right)=\infty, T C_{32}\left(T_{32}\right)=81.97423, T C_{33}\left(T_{33}\right)=\infty, T C_{41}\left(T_{41}\right)=\infty,
$$

$T C_{42}\left(T_{42}\right)=\infty$. Since $T_{32}>T_{d}$ and $N<\mu<M<T_{32}+N$, by Corollary 2(b)(ii), we know that the optimal solution is $T^{*}=T_{32}=0.23658$ years, and then $T C\left(T^{*}\right)=T C_{32}\left(T_{32}\right)=81.97423$. By (3), we have $Q^{*}=Q_{32}=24.59067$ units.

Example 2.3. Let $\mu=0.2$ years, we know that $N<M<\mu$. By (4), we have $T_{d}=0.19091$ years and by the above algorithm, we have

$$
T_{31}=0.22922, T_{32}=0.22946, T_{33}=0.24603, T_{41}=0.22611, T_{42}=0.23447
$$

and

$$
T C_{31}\left(T_{31}\right)=\infty, T C_{32}\left(T_{32}\right)=\infty, T C_{33}\left(T_{33}\right)=82.41147, T C_{41}\left(T_{41}\right)=\infty,
$$
$T C_{42}\left(T_{42}\right)=\infty$. Since $T_{32}>T_{d}$ and $N<M<\mu<T_{33}+N$, by Corollary 2(b) (iii), we know that the optimal solution is $T^{*}=T_{33}=0.24603$ years, and then $T C\left(T^{*}\right)=T C_{33}\left(T_{33}\right)=82.41147$. By (3), we have $Q^{*}=Q_{33}=26.06367$ units.

2) Let $M=1 / 3$ years, and $N=1 / 12$ years.

Example 2.4. Let $\mu=0.05$ years, we know that $\mu<N<M$. By (4), we have $T_{d}=0.19573$ years and by the above algorithm, we have

$$
T_{31}=0.20977, T_{32}=0.20953, T_{33}=0.22573, T_{41}=0.23617, T_{42}=0.23366
$$

and

$$
T C_{31}\left(T_{31}\right)=\infty, T C_{32}\left(T_{32}\right)=\infty, T C_{33}\left(T_{33}\right)=\infty, T C_{41}\left(T_{41}\right)=71.91272,
$$
$T C_{42}\left(T_{42}\right)=\infty$. Since $T_{41}>T_{d}$ and $\mu<N<T_{41}+N<M$, by Corollary 2(c)(i), we know that the optimal solution is $T^{*}=T_{41}=0.23617$ years, and then $T C\left(T^{*}\right)=T C_{41}\left(T_{41}\right)=71.91272$. By (3), we have $Q^{*}=Q_{41}=24.14471$ units.

Example 2.5. Let $\mu=0.1$ years, we know that $N<\mu<M$. By (4), we have $T_{d}=0.19286$ years and by the above algorithm, we have

$$
T_{31}=0.20641, T_{32}=0.20653, T_{33}=0.21635, T_{41}=0.23336, T_{42}=0.23459
$$

and

$$
T C_{31}\left(T_{31}\right)=\infty, T C_{32}\left(T_{32}\right)=\infty, T C_{33}\left(T_{33}\right)=\infty, T C_{41}\left(T_{41}\right)=\infty,
$$

$T C_{42}\left(T_{42}\right)=73.05178$. Since $T_{42}>T_{d}$ and $N<\mu<T_{42}+N<M$, by Corollary 2(c)(ii), we know that the optimal solution is $T^{*}=T_{42}=0.23459$ years, and 
then $T C\left(T^{*}\right)=T C_{42}\left(T_{42}\right)=73.05178$. By (3), we have $Q^{*}=Q_{42}=24.38186$ units.

From Table 1, some managerial insights can be obtained. In the case of $M<N$,

a) The retailer's total relevant cost $T C\left(T^{*}\right)$ increases as the predetermined order quantity $Q_{d}$ increase, and the constant rate of a period time $\mu$ increase. That is, if the predetermined order quantity $\left(Q_{d}\right)$ is larger and the period time $(\mu)$ is longer, then the total relevant cost $T C\left(T^{*}\right)$ for the retailer will be large.

b) As the order quantity is less than the predetermined quantity, $\left(T^{*}<T_{d}\right)$, the optimal replenishment cycle $T^{*}$ and the optimal order quantity $Q^{*}$ increase as the time point $(\mu)$ increases, while in the case, the order quantity is larger than the predetermined quantity $\left(T^{*}>T_{d}\right)$, the optimal replenishment cycle $T^{*}$ decreases as the time point $(\mu)$ increases.

In this case, if the upstream trade credit period is less than the downstream trade credit period, $(M<N)$, then the retailer need to pay more interest than earned, thus, the larger $Q_{d}$, and $\mu$ will cause the larger total relevant cost.

From Table 2, some managerial insights can be obtained. In the case of $M \geq N$,

a) The retailer's total relevant cost $T C\left(T^{*}\right)$ and the optimal order quantity $Q^{*}$ increase as the constant rate of a period time $(\mu)$ increases. That is, if the period time $(\mu)$ is longer, then it will cause the retailer's total relevant cost and the optimal order quantity to be larger.

b) The retailer's total relevant cost $T C\left(T^{*}\right)$ decreases as the upstream trade credit period $M$ increases, since the retailer may earn more interest than paid.

c) The larger the difference of $M-N$, (i.e., the shorter downstream trade credit period, and the longer upstream trade credit period), the less the retailer's total relevant cost is. That is, it's more profitable for the retailer.

Table 1. Summary on optimal solutions for Examples 1.1-1.5.

\begin{tabular}{cccccc}
\hline \multicolumn{5}{c}{$M<N$} \\
\hline Example & 1.1 & 1.2 & 1.3 & 1.4 & 1.5 \\
\hline$M$ & & & $1 / 12$ & \\
$N$ & & & $1 / 6$ & & \\
$Q_{d}$ & & 30 & & 20 & 0.2 \\
$\mu$ & 0.1 & 0.2 & 0.05 & 0.1 & 0.19091 \\
$T_{d}$ & 0.28810 & 0.28182 & 0.19573 & 0.19286 & 0.23191 \\
$T^{*}$ & 0.23986 & 0.24440 & 0.24312 & 0.23993 & 24.51676 \\
$Q^{*}$ & 24.93522 & 25.88441 & 24.8573 & 24.94312 & 86.95530 \\
$T C\left(T^{*}\right)$ & 88.36124 & 88.71856 & 84.79927 & 85.76229 & 3.2 .1 \\
& 3.1 & 3.1 & 3.2 .1 & 3.2 .1 & $T^{*}>T_{d}$ \\
Case & $T^{*}<T_{d}$ & $T^{*}<T_{d}$ & $T^{*}>T_{d}$ & $T^{*}>T_{d}$ & $M<\mu<T^{*}$ \\
& $\mu<N<T^{*}$ & $N<\mu<T^{*}$ & $\mu<M^{*}<T^{*}$ & $M<\mu<N<T^{*}$ & $M<\mu^{2}$ \\
Corollary & $1(\mathrm{a})$ & $1(\mathrm{~b})$ & $2(\mathrm{a})(\mathrm{i})$ & $2(\mathrm{a})(\mathrm{ii})$ & $2(\mathrm{a})(\mathrm{iii})$ \\
\hline
\end{tabular}


Table 2. Summary on optimal solutions for Examples 2.1-2.5.

\begin{tabular}{cccccc}
\hline \multicolumn{5}{c}{$M \geq N$} \\
Example & 2.1 & 2.2 & 2.3 & 2.4 & 2.5 \\
\hline$M$ & & $1 / 6$ & & & $1 / 3$ \\
$N$ & & & & \\
$Q_{d}$ & & & & \\
$\mu^{\prime}$ & & & & \\
$T_{d}$ & 0.05 & 0.1 & 0.2 & 0.05 & 0.1 \\
$T^{*}$ & 0.19573 & 0.19286 & 0.19091 & 0.19573 & 0.19286 \\
$Q^{*}$ & 0.23967 & 0.23658 & 0.24603 & 0.23617 & 0.23459 \\
$T C\left(T^{*}\right)$ & 24.50358 & 24.59067 & 26.06367 & 24.14471 & 24.38186 \\
& 81.06808 & 81.97423 & 82.41147 & 71.91272 & 73.05178 \\
& 3.2 .2 & 3.2 .2 & 3.2 .2 & 3.2 .3 & 3.2 .3 \\
Case & $T^{*}>T_{d}$ & $T^{*}>T_{d}$ & $T^{*}>T_{d}$ & $T^{*}>T_{d}$ & $T^{*}>T_{d}$ \\
& $\mu<N<M$ & $N<\mu<M$ & $N<M<\mu$ & $\mu<N<M$ & $N<\mu<M$ \\
& $M<T^{*}+N$ & $M<T^{*}+N$ & $M<T^{*}+N$ & $M>T^{*}+N$ & $M>T^{*}+N$ \\
Corollary & $2(\mathrm{~b})(\mathrm{i})$ & $2(\mathrm{~b})(\mathrm{ii})$ & $2(\mathrm{~b})(\mathrm{iii})$ & $2(\mathrm{c})(\mathrm{i})$ & $2(\mathrm{c})(\mathrm{ii})$ \\
\hline
\end{tabular}

In summary, the longer the upstream trade credit period $M$, the less the predetermined order quantity $Q_{d}$, and the less the constant rate of a period time $\mu$, will cause the less the retailer's total relevant cost. However, the larger the downstream trade credit period $N$, will cause the larger the retailer's total relevant cost.

\section{Conclusions}

In this study, we develop an inventory model in a supply chain with ramp-type demand and trade credit financing linked to order quantity. The supplier offers a permissible delay linked to order quantity, while the retailer also provides a downstream trade credit period to its customers. We have obtained some theoretical results to characterize the optimal solutions and presented several numerical examples to illustrate the proposed models. The results reveal that 1) $T^{*}<T_{d}$ will cause more retailer's total relevant cost than other cases, since there is no upstream trade credit period allowed, the retailer need to pay more interest than earned. 2) $T^{*}>T_{d}$ and $N<M, M>T^{*}+N$ will cause less retailer's total relevant cost than the others, since the retailer can earn more interest than paid. It's more profitable for the retailer in such case. 3) The retailer's total relevant cost increase as any one of the parameter values $\mu, Q_{d}, N$ increases, while decreases as $M$ increases. Thus, if upstream trade credit period is longer, then the retailer's total relevant cost will be less, it's more benefit for the retailer.

The model can be extended in several ways, for example, we may consider the item with a constant deterioration rate. Also, we can extend the model to allow for shortages and partial backlogging. Finally, we could add the pricing, advertising and quality strategies into consideration. 


\section{Conflicts of Interest}

The author declares no conflicts of interest regarding the publication of this paper.

\section{References}

[1] Wu, K.S. (2001) An EOQ Inventory Model for Items with Weibull Distribution Deterioration, Ramp Type Demand Rate and Partial Backlogging. Production Planning \& Control, 12, 787-793. https://doi.org/10.1080/09537280110051819

[2] Manna, S.K. and Chaudhrui, K.S. (2006) An EOQ Model with Ramp Type Demand Rate, Time Dependent Deterioration Rate, Unit Production Cost and Shortages. European Journal of Operational Research, 171, 557-566. https://doi.org/10.1016/j.ejor.2004.08.041

[3] Agrawal, S., Banerjee, S., and Papachristos, S. (2013) Inventory Model with Deteriorating Items, Ramp-Type Demand and Partially Backlogged Shortages for a Two Warehouse System. Applied Mathematical Modelling, 37, 8912-8929. https://doi.org/10.1016/j.apm.2013.04.026

[4] Deng, P.S. (2005) Improved Inventory Models with Ramp Type Demand and Weibull Deterioration. International Journal of Information and Management Sciences, 16, 79-86.

[5] Deng, P.S., Lin, R.H.J. and Chu, P. (2007) A Note on the Inventory Models for Deteriorating Items with Ramp Type Demand Rate. European Journal of Operational Research, 178, 112-120. https://doi.org/10.1016/j.ejor.2006.01.028

[6] Panda, S., Saha, S. and Basu, M. (2007) An EOQ Model with Generalized RampType Demand and Weibull Distribution Deterioration. Asia-Pacific Journal of $O p$ erational Research, 24, 93-109. https://doi.org/10.1142/S0217595907001152

[7] Panda, S., Senapati, S. and Basu, M. (2008) Optimal Replenishment Policy for Perishable Seasonal Products in a Season with Ramp-Type Dependent Demand. Computer \& Industrial Engineering, 54, 301-314. https://doi.org/10.1016/j.cie.2007.07.011

[8] Skouri, K., Konstantaras, I., Papachristos, S. and Ganas, I. (2009) Inventory Models with Ramp Type Demand Rate, Partial Backlogging and Weibull Deterioration Rate. European Journal of Operational Research, 192, 79-92. https://doi.org/10.1016/j.ejor.2007.09.003

[9] Skouri, K., Konstantaras, I. and Manna, S.K. (2011) Inventory Models with Ramp Type Demand Rate, Time Dependent Deterioration Rate, Unit Production Cost and Shortages. Annals of Operations Research, 191, 73-95. https://doi.org/10.1007/s10479-011-0984-2

[10] Goyal, S.K. (1985) Economic Order Quantity under Conditions of Permissible Delay in Payments. Journal of the Operational Research Society, 36, 335-338. https://doi.org/10.1057/jors.1985.56

[11] Shah, N.H. (1993) Probabilistic Time-Scheduling Model for an Exponentially Decaying Inventory When Delay in Payments Is Permissible. International Journal of Production Economics, 32, 77-82. https://doi.org/10.1016/0925-5273(93)90009-A

[12] Aggarwal, S.P. and Jaggi, C.K. (1995) Ordering Policies of Deteriorating Items under Permissible Delay in Payments. Journal of the Operational Research Society, 46, 658-662. https://doi.org/10.1057/jors.1995.90

[13] Jamal, A.M.M., Sarker, B.R. and Wang, S. (1997) An Ordering Policy for Deteriorating Items with Allowable Shortages and Permissible Delay in Payments. Journal 
of the Operational Research Society, 48, 826-833. https://doi.org/10.1057/palgrave.jors.2600428

[14] Teng, J.T. (2002) On the Economic Order Quantity under Conditions of Permissible Delay in Payments. Journal of the Operational Research Society, 53, 915-918. https://doi.org/10.1057/palgrave.jors.2601410

[15] Skouri, K., Konstantaras, I., Papachristos, S. and Teng, J.T. (2011) Supply Chain Models for Deteriorating Products with Ramp Type Demand Rate under Permissible Delay in Payments. Expert Systems with Applications, 38, 14861-14869. https://doi.org/10.1016/j.eswa.2011.05.061

[16] Teng, J.T., Min, J. and Pan, Q.H. (2012) Economic Order Quantity Model with Trade Credit Financing for Non-Decreasing Demand. Omega, 40, 328-335. https://doi.org/10.1016/j.omega.2011.08.001

[17] Huang, Y.F. (2003) Optimal Retailer's Ordering Policies in the EOQ Model under Trade Credit Financing. Journal of the Operational Research Society, 54, 1011-1015. https://doi.org/10.1057/palgrave.jors.2601588

[18] Teng, J.T. and Goyal, S.K. (2007) Optimal Ordering Policies for a Retailer in a Supply Chain with Up-Stream and Down-Stream Trade Credits. Journal of Operational Research Society, 58, 1252-1255. https://doi.org/10.1057/palgrave.jors.2601588

[19] Teng, J.T., Krommyda, I.P., Skouri, K. and Lou, K.R. (2011) A Comprehensive Extension of Optimal Ordering Policy for Stock-Dependent Demand under Progressive Payment Scheme. European Journal of Operational Research, 215, 97-104. https://doi.org/10.1016/j.ejor.2011.05.056

[20] Chen, S.C. and Teng, J.T. (2014) Retailer's Optimal Ordering Policy for Deteriorating Items with Maximum Lifetime under Supplier's Trade Credit Financing. $A p$ plied Mathematical Modelling, 38, 4049-4061. https://doi.org/10.1016/j.apm.2013.11.056

[21] Chen, S.C. and Teng, J.T. (2015) Inventory and Credit Decisions for Time-Varying Deteriorating Items with Up-Stream and Down-Stream Trade Credit Financing by Discounted Cash Flow Analysis. European Journal of Operational Research, 243, 566-575. https://doi.org/10.1016/j.ejor.2014.12.007

[22] Shah, N.H. (2017) Three-Layered Integrated Inventory Model for Deteriorating Items with Quadratic Demand and Two-Level Trade Credit Financing. International Journal of Systems Science: Operation Logistics, 4, 85-91.

[23] Rameswari, M. and Uthayakumar, R. (2018) An Integrated Inventory Model for Deteriorating Items with Price-Dependent Demand under Two-Level Trade Credit Policy. International Journal of Systems Science: Operation Logistics, 5, 253-267.

[24] Goyal, S.K., Teng, J.T. and Chang, C.T. (2007) Optimal Ordering Policies When the Supplier Provides a Progressive Interest-Payable Scheme. European Journal of Operational Research, 179, 404-413. https://doi.org/10.1016/j.ejor.2006.03.037

[25] Huang, Y.F. and Hsu, K.H. (2008) An EOQ Model under Retailer Partial Trade Credit Policy in Supply Chain. International Journal of Production Economics, 112, 655-664. https://doi.org/10.1016/j.ijpe.2007.05.014

[26] Min, J., Zhou, Y.W. and Zhao, J. (2010) An Inventory Model for Deteriorating Items under Stock-Dependent Demand and Two Level Trade Credit. Applied Mathematical Modelling, 34, 3273-3285. https://doi.org/10.1016/j.apm.2010.02.019

[27] Chang, C.T., Ouyang, L.Y. and Teng, J.T. (2003) An EOQ Model for Deteriorating Items under Supplier Credits Linked to Order Quantity. Applied Mathematical Modelling, 27, 983-996. https://doi.org/10.1016/S0307-904X(03)00131-8 
[28] Chung, K.J. and Liao, J.J. (2004) Lot-Sizing Decisions under Trade Credit Depending on the Ordering Quantity. Computers Operations Research, 31, 909-928. https://doi.org/10.1016/S0305-0548(03)00043-1

[29] Ouyang, L.Y., Teng, J.T., Goyal, S.K. and Yang, C.T. (2009) An Economic Order Quantity for Deteriorating Items with Partially Permissible Delay in Payments Linked to Order Quantity. European Journal of Operational Research, 194, 418-431. https://doi.org/10.1016/j.ejor.2007.12.018

[30] Kreng, V.B. and Tan, S.J. (2010) The Optimal Replenishment Decision under Two Levels of Trade Credit Policy Depending on the Order Quantity. Expert Systems with Applications, 37, 5514-5522. https://doi.org/10.1016/j.eswa.2009.12.014

[31] Teng, J.T., Yang, H.L. and Chern, M.S. (2013) An Inventory Model for Increasing Demand under Two Levels of Trade Credit Linked to Order Quantity. Applied Mathematical Modelling, 37, 7624-7632. https://doi.org/10.1016/j.apm.2013.02.009

[32] Shah, N.H. and Cardenas-Barrón, L.E. (2015) Retailer's Decision for Ordering and Credit Policies for Deteriorating Items When a Supplier Offers order-Linked Credit or Cash Discount. Applied Mathematics and Computation, 259, 569-578. https://doi.org/10.1016/j.amc.2015.03.010

[33] Ting, P.S. (2015) Comments on the EOQ Model for Deteriorating Items with Conditional Trade Credit Linked to Order Quantity. European Journal of Operational Research, 246, 108-118. https://doi.org/10.1016/j.ejor.2015.04.046 


\section{Appendix: Proof of Theorem 1}

For $T C_{11}$, from (14.1), let

$$
F(T)=\frac{\mathrm{d} T C_{11}}{\mathrm{~d} T}=\left\{h(a+b \mu) T+c I_{p}(a+b \mu)(T+N)-T C_{11}\right\} / T=0 .
$$

Differentiating $F(T)$ with respect to $T$, we have

$$
\left.\frac{\mathrm{d} F(T)}{\mathrm{d} T}\right|_{\frac{\mathrm{d} T C_{1 j}}{\mathrm{~d} T}=0}=\left.\frac{\mathrm{d}^{2} T C_{1 j}}{\mathrm{~d} T^{2}}\right|_{\frac{\mathrm{d} T C_{1 j}}{\mathrm{~d} T}=0}=\left(h+c I_{p}\right)(a+b \mu) / T>0 .
$$

This implies that $F(T)$ is an increasing function of $T$. Furthermore,

$$
\lim _{T \rightarrow 0} F(T)=\lim _{T \rightarrow 0}\left\{h(a+b \mu) T+c I_{p}(a+b \mu)(T+N)\right\} / T-\lim _{T \rightarrow 0} T C_{11} / T=-\infty<0,
$$

since $\lim _{T \rightarrow 0} T C_{11} / T=\infty$. And

$$
\lim _{T \rightarrow \infty} F(T)=\left(h+c I_{p}\right)(a+b \mu)-\lim _{T \rightarrow \infty} T C_{11} / T=\left(h+c I_{p}\right)(a+b \mu) / 2>0,
$$

since

$$
\begin{aligned}
\lim _{T \rightarrow \infty} T C_{11} / T \\
=\lim _{T \rightarrow \infty}\left\{A+h\left[\frac{a \mu^{2}}{2}+\frac{b \mu^{3}}{3}+\frac{(a+b \mu)\left(T^{2}-\mu^{2}\right)}{2}\right]\right. \\
\left.+c I_{p}\left[\left(a \mu+\frac{b \mu^{2}}{2}\right) \mu+(a+b \mu)\left(T N-\mu^{2}\right)+\frac{a+b \mu}{2} T^{2}\right]\right\} / T^{2} \\
=\lim _{T \rightarrow \infty}\left\{h(a+b \mu) T+c I_{p}(a+b \mu)(T+N)\right\} /(2 T) \quad \text { (By L'Hospital's Rule) } \\
=\left(h+c I_{p}\right)(a+b \mu) / 2
\end{aligned}
$$

Therefore, there exists an unique solution such that $F(T)=0$. From (15), we know that the solution which minimizes $T C_{11}$. The other cases can also be proved by the similar way. Thus, the Theorem is proved. 